\title{
Dimensional REDUCTION OF THE ABJM MODEL
}

\author{
Horatiu Nastase ${ }^{a, *}$ and Constantinos Papageorgakis ${ }^{b, \dagger}$ \\ ${ }^{a}$ Instituto de Física Teórica, UNESP-Universidade Estadual Paulista \\ R. Dr. Bento T. Ferraz 271, Bl. II, Sao Paulo 01140-070, SP, Brazil \\ ${ }^{b}$ Department of Mathematics, King's College London \\ The Strand, London WC2R 2LS, UK
}

\begin{abstract}
We dimensionally reduce the ABJM model, obtaining a two-dimensional theory that can be thought of as a 'master action'. This encodes information about both T- and S-duality, i.e. describes fundamental (F1) and D-strings (D1) in 9 and 10 dimensions. The Higgsed theory at large VEV, $\tilde{v}$, and large $k$ yields D1brane actions in $9 \mathrm{~d}$ and $10 \mathrm{~d}$, depending on which auxiliary fields are integrated out. For $N=1$ there is a map to a Green-Schwarz string wrapping a nontrivial circle in $\mathbb{C}^{4} / \mathbb{Z}_{k}$.
\end{abstract}

\footnotetext{
${ }^{*}$ E-mail address: nastase@ift.unesp.br

${ }^{\dagger}$ E-mail address: costis.papageorgakis@kcl.ac.uk
} 


\section{Introduction}

The Aharony-Bergman-Jafferis-Maldacena (ABJM) model [1] has received a lot of attention lately, as it captures the dynamics of multiple M2-branes in a particular M-theory background. Whereas the Bergshoeff-Sezgin-Townsend (BST) action [2] for a single membrane has no gauge fields, is generically nonconformal and contains the membrane tension parameter $T_{2}$, the ABJM theory is a $\mathrm{U}(N) \times \mathrm{U}(N)$, conformal, Chern-Simons-matter gauge theory at level $k$ with $\mathcal{N}=6$ supersymmetry, corresponding to the IR limit of $N$ M2-branes on a $\mathbb{C}^{4} / \mathbb{Z}_{k}$ singularity. The interest in the ABJM model arises both from being the first example of a multiple membrane theory, as well as from the fact that it provides a new direction for $\mathrm{AdS}_{4} / \mathrm{CFT}_{3}$, being dual to string theory in the $\mathrm{AdS}_{4} \times \mathbb{C P}^{3}$ background at large $k .^{1}$

It is well known that, after double dimensional reduction, the BST action yields the Green-Schwarz (GS) description of a fundamental string (F1) [3]. One could also consider reducing on two, instead of one, circles: The M2 can first be compactified on a worldvolume direction down to an F1-string in 10d and then on a transverse direction to a type IIA F1 in 9d. But if instead one first reduces on a transverse and then on a parallel direction, the result is a type IIA D1 in 9d. The two procedures are related by an S-duality transformation, after also having implemented a T-duality to IIB configurations in 10d.

In the same spirit it is natural to expect that the dimensionally-reduced ABJM model should also be related to a multiple fundamental string action. An immediate problem with that assumption is that there exists no such known example. Moreover one would not expect it to contain gauge fields, obtained from the ABJM Chern-Simons gauge fields, which would be more in line with having a theory of D1-branes. Finally, there is also an additional effective compactification occurring for large $k$ in the spirit of $[4,5]$, which however seems to commute with the naïve dimensional reduction and hence contradicts the intuition of the two-circle compactification described above.

In this note we will analyse the dimensional reduction of the ABJM model in more detail and argue that the resulting theory can be interpreted as a 'master action' that encodes information for both T- and S-duality: Depending on the variables used and the energy regime one is interested in, we obtain either multiple F- or D-strings in $9 \mathrm{~d}$ or $10 \mathrm{~d}$. There exists some related work [6, 7], particularly pertaining to the dimensional reduction of the Bagger-Lambert-Gustavsson (BLG) model [8, 9], although in our opinion the interpretation of the final action has not been explored in the same fashion.

The rest of this paper is organised as follows. We perform the dimensional reduction, showing in Section 2.1 that in the presence of a large VEV the Higgsed theory reduces to

\footnotetext{
${ }^{1}$ The near-horizon geometry for M2-branes on $\mathbb{C}^{4} / \mathbb{Z}_{k}$ is $\mathrm{AdS}_{4} \times S^{7} / \mathbb{Z}_{k}$. The orbifold action is such that $S^{1} / \mathbb{Z}_{k} \hookrightarrow S^{7} / \mathbb{Z}_{k} \stackrel{\pi}{\rightarrow} \mathbb{C P}^{3}$, so the geometry reduces to $\mathrm{AdS}_{4} \times \mathbb{C P}^{3}$ in the large- $k$ limit.
} 
either the D1-brane action in $9 \mathrm{~d}$ or the D1-brane action in $10 \mathrm{~d}$, with the two related by T-duality. We also calculate the action for $N=1$ in terms of a particular set of variables. In Section 3 we proceed to exhibit a transformation that turns the $N=1$ ABJM model into the usual BST action. The same transformation turns our reduced action for $N=1$ into a GS-string action. We finally interpret the results, showing in particular that the latter correctly corresponds to a string in a $\mathbb{C}^{4} / \mathbb{Z}_{k}$ background for general parameters.

\section{Dimensional reduction}

The ABJM action [1], corresponding to the IR limit of $N$ M2-branes at an $\mathbb{R}^{2,1} \times \mathbb{C}^{4} / \mathbb{Z}_{k}$ singularity, is given by

$$
\begin{aligned}
S_{A B J M}= & \int d^{3} x\left[\frac{k}{4 \pi} \epsilon^{\mu \nu \lambda} \operatorname{Tr}\left(A_{\mu}^{(1)} \partial_{\nu} A_{\lambda}^{(1)}+\frac{2 i}{3} A_{\mu}^{(1)} A_{\nu}^{(1)} A_{\lambda}^{(1)}-A_{\mu}^{(2)} \partial_{\nu} A_{\lambda}^{(2)}-\frac{2 i}{3} A_{\mu}^{(2)} A_{\nu}^{(2)} A_{\lambda}^{(2)}\right)\right. \\
& -\operatorname{Tr}\left(D_{\mu} C_{I}^{\dagger} D^{\mu} C^{I}\right)-i \operatorname{Tr}\left(\psi^{I \dagger} \gamma^{\mu} D_{\mu} \psi_{I}\right) \\
& +\frac{4 \pi^{2}}{3 k^{2}} \operatorname{Tr}\left(C^{I} C_{I}^{\dagger} C^{J} C_{J}^{\dagger} C^{K} C_{K}^{\dagger}+C_{I}^{\dagger} C^{I} C_{J}^{\dagger} C^{J} C_{K}^{\dagger} C^{K}\right. \\
& \left.+4 C^{I} C_{J}^{\dagger} C^{K} C_{I}^{\dagger} C^{J} C_{K}^{\dagger}-6 C^{I} C_{J}^{\dagger} C^{J} C_{I}^{\dagger} C^{K} C_{K}^{\dagger}\right) \\
& +\frac{2 \pi i}{k} \operatorname{Tr}\left(C_{I}^{\dagger} C^{I} \psi^{J \dagger} \psi_{J}-\psi^{\dagger J} C^{I} C_{I}^{\dagger} \psi_{J}-2 C_{I}^{\dagger} C^{J} \psi^{\dagger I} \psi_{J}+2 \psi^{\dagger J} C^{I} C_{J}^{\dagger} \psi_{I}\right. \\
& \left.\left.+\epsilon^{I J K L} \psi_{I} C_{J}^{\dagger} \psi_{K} C_{L}^{\dagger}-\epsilon_{I J K L} \psi^{\dagger I} C^{J} \psi^{\dagger K} C^{L}\right)\right]
\end{aligned}
$$

where the Lorentz index $\mu=0,1,2$ and the R-symmetry index $I=1, \ldots, 4$ in $\mathrm{SU}(4)$.

There also exists a maximally supersymmetric massive deformation $[10,11]$ where one splits the scalars as $C^{I}=\left(R^{\alpha}, Q^{\alpha}\right)$, with $\alpha=1,2$. Then the mass deformation changes the potential to

$$
V=\left|M^{\alpha}\right|^{2}+\left|N^{\alpha}\right|^{2}
$$

where

$$
\begin{aligned}
M^{\alpha} & =\mu Q^{\alpha}+\frac{2 \pi}{k}\left(2 Q^{[\alpha} Q_{\beta}^{\dagger} Q^{\beta]}+R^{\beta} R_{\beta}^{\dagger} Q^{\alpha}-Q^{\alpha} R_{\beta}^{\dagger} R^{\beta}+2 Q^{\beta} R_{\beta}^{\dagger} R^{\alpha}\right) \\
N^{\alpha} & =-\mu R^{\alpha}+\frac{2 \pi}{k}\left(2 R^{[\alpha} R_{\beta}^{\dagger} R^{\beta]}+Q^{\beta} Q_{\beta}^{\dagger} R^{\alpha}-R^{\alpha} Q_{\beta}^{\dagger} Q^{\beta}+2 R^{\beta} Q_{\beta}^{\dagger} Q^{\alpha}\right)
\end{aligned}
$$

In addition, the potential involves a mass term $\mu$ for the fermions.

In order to dimensionally reduce the above on a circle of radius $R$, we choose the standard ansatz by dropping the dependence of all fields on the circle direction $y$, where we split $x^{\mu}=x^{i}, y$, with $i=0,1 .^{2}$ We further need to rescale the fields by powers of the

\footnotetext{
${ }^{2}$ Since we are compactifying on a circle by keeping all the fields and just dropping the circle dependence, we have automatically obtained a consistent truncation: The spherical harmonics are trivial (Fourier modes), so there are no terms linear in the massive (dropped) fields in the action (a massive Fourier mode $e^{2 \pi i n y / R}$ needs at least another massive mode $e^{-2 \pi i n / R}$ to give a nonzero result after integration).
} 
radius $R$ to obtain the canonical dimension in $2 \mathrm{~d}$. This leads to the following ansatz

$$
C^{I}=\frac{1}{\sqrt{R}} \tilde{C}^{I}(\vec{x}) ; \quad \psi=\frac{1}{\sqrt{R}} \tilde{\psi}(\vec{x}) ; \quad A_{i}^{(1,2)}=A_{i}^{(1,2)}(\vec{x}) ; \quad A_{y}^{(1,2)}=\frac{1}{R} \phi_{1,2}(\vec{x}) .
$$

The covariant derivatives are

$$
\begin{aligned}
& D_{i} C^{I}=\partial_{i} C^{I}-i A_{i}^{(1)} C^{I}+i C^{I} A_{i}^{(2)} \\
& D_{y} C^{I}=i \frac{1}{R}\left(C^{I} \phi_{2}-\phi_{1} C^{I}\right)
\end{aligned}
$$

and similarly for the fermions. From the 'pure' (undeformed) ABJM action (2.1) we then get the dimensionally reduced action

$$
\begin{aligned}
S_{2 d}= & \int d^{2} x\left[\frac{k}{4 \pi} \epsilon^{i j} \operatorname{Tr}\left(\phi_{1} F_{i j}^{(1)}-\phi_{2} F_{i j}^{(2)}\right)-\operatorname{Tr}\left(D_{i} \tilde{C}_{I}^{\dagger} D^{i} \tilde{C}^{I}\right)-i \operatorname{Tr}\left(\tilde{\psi}^{I \dagger} \gamma^{i} D_{i} \tilde{\psi}_{I}\right)\right. \\
& +\frac{4 \pi^{2}}{3 k^{2} R^{2}} \operatorname{Tr}\left(\tilde{C}^{I} \tilde{C}_{I}^{\dagger} \tilde{C}^{J} \tilde{C}_{J}^{\dagger} \tilde{C}^{K} \tilde{C}_{K}^{\dagger}+\tilde{C}_{I}^{\dagger} \tilde{C}^{I} \tilde{C}_{J}^{\dagger} \tilde{C}^{J} \tilde{C}_{K}^{\dagger} \tilde{C}^{K}\right. \\
& \left.+4 \tilde{C}^{I} \tilde{C}_{J}^{\dagger} \tilde{C}^{K} \tilde{C}_{I}^{\dagger} \tilde{C}^{J} \tilde{C}_{K}^{\dagger}-6 \tilde{C}^{I} \tilde{C}_{J}^{\dagger} \tilde{C}^{J} \tilde{C}_{I}^{\dagger} \tilde{C}^{K} \tilde{C}_{K}^{\dagger}\right) \\
& +\frac{2 \pi i}{k R} \operatorname{Tr}\left(\tilde{C}_{I}^{\dagger} \tilde{C}^{I} \tilde{\psi}^{J \dagger} \tilde{\psi}_{J}-\tilde{\psi}^{\dagger J} \tilde{C}^{I} \tilde{C}_{I}^{\dagger} \tilde{\psi}_{J}-2 \tilde{C}_{I}^{\dagger} \tilde{C}^{J} \tilde{\psi}^{\dagger I} \tilde{\psi}_{J}+2 \tilde{\psi}^{\dagger J} \tilde{C}^{I} \tilde{C}_{J}^{\dagger} \tilde{\psi}_{I}\right. \\
& \left.+\epsilon^{I J K} \tilde{\psi}_{I} \tilde{C}_{J}^{\dagger} \tilde{\psi}_{K} \tilde{C}_{L}^{\dagger}-\epsilon_{I J K L} \tilde{\psi}^{\dagger I} \tilde{C}^{J} \tilde{\psi}^{\dagger} \tilde{C}^{L}\right) \\
& +R^{-2} \operatorname{Tr}\left(\left(\tilde{C}^{I} \phi_{2}-\phi_{1} \tilde{C}^{I}\right)\left(\tilde{C}_{I}^{\dagger} \phi_{1}-\phi_{2} \tilde{C}_{I}^{\dagger}\right)\right) \\
& \left.+R^{-1} \operatorname{Tr}\left(\tilde{\psi}^{I \dagger} \gamma_{3}\left(\tilde{\psi}_{I} \phi_{2}-\phi_{1} \tilde{\psi}_{I}\right)\right)\right]
\end{aligned}
$$

where

$$
F_{i j}^{(1,2)}=\partial_{i} A_{j}^{(1,2)}-\partial_{j} A_{i}^{(1,2)}+i A_{i}^{(1,2)} A_{j}^{(1,2)}-i A_{j}^{(1,2)} A_{i}^{(1,2)} .
$$

The fields $\phi_{1,2}$ are auxiliary (nonpropagating) and as a result could be eliminated from the action.

In order to get a feeling for the general case, we first set $\phi_{2}=0$. This is not a solution, i.e. a consistent truncation, so the following is just for purposes of illustration. The bosonic $\phi_{1}$ action reduces to

$$
\int d^{2} x\left[\frac{\sqrt{2}}{2 g R} \operatorname{Tr}\left(\epsilon^{i j} \phi_{1} F_{i j}^{(1)}\right)-R^{-2} \operatorname{Tr}\left(\left(\phi_{1}\right)^{2} \tilde{C}^{I} \tilde{C}_{I}^{\dagger}\right)\right],
$$

where we have defined

$$
g=\frac{2 \pi \sqrt{2}}{k R}
$$

Solving for $\phi_{1}$

$$
\phi_{1}=\frac{R \sqrt{2}}{4 g} \epsilon^{i j} F_{i j}^{(1)}\left(\tilde{C}^{I} \tilde{C}_{I}^{\dagger}\right)^{-1}
$$

and replacing in (2.8), while using that in $2 \mathrm{~d}\left(\epsilon^{i j} F_{i j}\right)^{2}=-2 F_{i j} F^{i j}$, we obtain the kinetic term

$$
-\frac{1}{4 g^{2}} \int d^{2} x \operatorname{Tr}\left[F_{i j}^{(1)} F^{(1) i j}\left(\tilde{C}^{I} \tilde{C}_{I}^{\dagger}\right)^{-1}\right]
$$


This is the standard kinetic term for the gauge field $A_{i}^{(1)}$, with a nonpolynomial scalar field dressing-factor.

However, when we include $\phi_{2}$, we get the equations

$$
\begin{aligned}
& \frac{R \sqrt{2} \epsilon^{i j} F_{i j}^{(1)}}{2 g}=2 \phi_{1}\left(\tilde{C}^{I} \tilde{C}_{I}^{\dagger}\right)-2 C^{I} \phi_{2} \tilde{C}_{I}^{\dagger}-R \tilde{\psi}_{I \alpha}\left(\tilde{\psi}^{I \dagger} \gamma_{3}\right)^{\alpha} \\
& -\frac{R \sqrt{2} \epsilon^{i j} F_{i j}^{(2)}}{2 g}=2 \phi_{2}\left(\tilde{C}_{I}^{\dagger} \tilde{C}^{I}\right)-2 C_{I}^{\dagger} \phi_{1} \tilde{C}^{I}-R\left(\tilde{\psi}^{I \dagger} \gamma_{3}\right)^{\alpha} \tilde{\psi}_{I \alpha}
\end{aligned}
$$

where we have explicitly written out the spinor indices $\alpha=1,2$. From the above one can derive an equation for $\phi_{1}$

$$
\begin{aligned}
\phi_{1}\left(\tilde{C}^{I} \tilde{C}_{I}^{\dagger}\right)-\tilde{C}^{K} \tilde{C}_{J}^{\dagger} \phi_{1} \tilde{C}^{J}\left(\tilde{C}_{I}^{\dagger} \tilde{C}^{I}\right)^{-1} \tilde{C}_{K}^{\dagger}= & \frac{R \sqrt{2}}{4 g} \epsilon_{i j}\left[F_{i j}^{(1)}-\tilde{C}^{K} F_{i j}^{(2)}\left(\tilde{C}_{I}^{\dagger} \tilde{C}^{I}\right)^{-1} \tilde{C}_{K}^{\dagger}\right] \\
& -\frac{R}{2} \tilde{C}^{K}\left(\tilde{\psi}^{J \dagger} \gamma_{3}\right)^{\alpha} \tilde{\psi}_{J \alpha}\left(\tilde{C}_{I}^{\dagger} \tilde{C}^{I}\right)^{-1} \tilde{C}_{K}^{\dagger} \\
& +\frac{R}{2} \tilde{\psi}_{I \alpha}\left(\tilde{\psi}^{I \dagger} \gamma_{3}\right)^{\alpha}
\end{aligned}
$$

that we cannot solve further. In principle, the solutions for $\phi_{1}, \phi_{2}$ should be then substituted back into the action

$$
\begin{array}{rl}
S_{2 d}=\int d^{2} & x\left[\frac{\sqrt{2}}{2 g R} \operatorname{Tr}\left(\phi_{1} \epsilon^{i j} F_{i j}^{(1)}-\phi_{2} \epsilon^{i j} F_{i j}^{(2)}\right)\right. \\
& -R^{-2} \operatorname{Tr}\left(\left(\phi_{1}\right)^{2} \tilde{C}^{I} \tilde{C}_{I}^{\dagger}+\left(\phi_{2}\right)^{2} \tilde{C}_{I}^{\dagger} \tilde{C}^{I}-2 \phi_{1} \tilde{C}^{I} \phi_{2} \tilde{C}_{I}^{\dagger}\right) \\
+ & \left.R^{-1} \operatorname{Tr}\left(\phi_{2} \tilde{\psi}^{I \dagger} \gamma_{3} \tilde{\psi}_{I}+\phi_{1} \tilde{\psi}_{I \alpha}\left(\tilde{\psi}^{I \dagger} \gamma_{3}\right)^{\alpha}\right)\right]
\end{array}
$$

and added to the $\phi_{i}$-independent part.

Note that the fields are massless, but the action is nonconformal since $g$ has dimensions of mass, as expected for the Yang-Mills coupling in 2d. It is also important to mention that the gauge fields that have been obtained are still nonpropagating, as the YM kinetic term in $2 \mathrm{~d}$ has $d-2=0$ degrees of freedom, and thus there is no contradiction with the counting of degrees of freedom before and after the reduction (the scalars remain scalars and the fermions do not lose degrees of freedom when going from $3 \mathrm{~d}$ to $2 \mathrm{~d}$ ).

Dimensionally reducing the mass-deformed ABJM theory is trivial: The mass deformation only affects the scalar potential and gives mass to the fermions, so these terms remain unaffected by going down to 2d. Similarly, the gauge field kinetic terms are the same as those for the undeformed ABJM theory, except for the fact that the supersymmetric vacuum is now the fuzzy sphere as in [11-14].

\subsection{Higgsing the reduced theory}

We next investigate the vacuum structure of the $2 \mathrm{~d}$ theory. The $\operatorname{VEV}\left\langle\tilde{C}^{I}\right\rangle=\tilde{v} \delta_{1}^{I} \mathbb{1}_{N \times N}$, with the rest of the fields set to zero, is a solution of the equations of motion. Expanding 
the theory (2.6) around this vacuum, and fixing for the moment the scalars to their VEV values, we obtain

$$
S=\int d^{2} x\left[-i \operatorname{Tr}\left(\tilde{\psi}^{I \dagger} \gamma^{i} D_{i} \tilde{\psi}_{I}\right)-\tilde{v}^{2} \operatorname{Tr}\left(A_{i}^{(1)}-A_{i}^{(2)}\right)^{2}\right]+S_{\phi},
$$

where

$$
\begin{array}{r}
S_{\phi}=\int d^{2} x\left[\frac{\sqrt{2}}{2 g R} \operatorname{Tr}\left(\phi_{1} \epsilon^{i j} F_{i j}^{(1)}-\phi_{2} \epsilon^{i j} F_{i j}^{(2)}\right)+R^{-1} \operatorname{Tr}\left(\phi_{2} \tilde{\psi}^{I \dagger} \gamma_{3} \tilde{\psi}_{I}+\phi_{1} \tilde{\psi}_{I \alpha}\left(\tilde{\psi}^{I \dagger} \gamma_{3}\right)^{\alpha}\right)\right. \\
\left.-\tilde{v}^{2} R^{-2} \operatorname{Tr}\left(\phi_{1}-\phi_{2}\right)^{2}\right] .
\end{array}
$$

Varying with respect to $\phi_{1}, \phi_{2}$ we then get the following constraints:

$$
\begin{aligned}
\phi_{1}-\phi_{2} & =\frac{R \sqrt{2}}{4 \tilde{v}^{2} g} \epsilon^{i j} F_{i j}^{(1)}+\frac{R}{2 \tilde{v}^{2}} \tilde{\psi}_{I \alpha}\left(\tilde{\psi}^{I \dagger} \gamma_{3}\right)^{\alpha} \\
\epsilon^{i j} F_{i j}^{(2)} & =\epsilon^{i j} F_{i j}^{(1)}+\sqrt{2} g\left(\tilde{\psi}_{I \alpha}\left(\tilde{\psi}^{I \dagger} \gamma_{3}\right)^{\alpha}+\tilde{\psi}^{I \dagger} \gamma_{3} \psi_{I}\right) .
\end{aligned}
$$

This implies that $F_{i j}^{(2)}$ is determined in terms of $F_{i j}^{(1)}$, as is $\phi_{1}-\phi_{2}$, although $\phi_{1}+\phi_{2}$ is still free. Nevertheless, substituting back in the auxiliary field action we get that $\phi_{1}+\phi_{2}$ also disappears from the action to give

$$
S=\int d^{2} x\left[-i \operatorname{Tr}\left(\tilde{\psi}^{I \dagger} \gamma^{i} D_{i} \tilde{\psi}_{I}\right)-\tilde{v}^{2} \operatorname{Tr}\left(A_{i}^{(1)}-A_{i}^{(2)}\right)^{2}\right]+S_{\phi},
$$

with

$S_{\phi}=\int d^{3} x\left[-\frac{1}{4 g^{2} \tilde{v}^{2}} \operatorname{Tr}\left(F_{i j}^{(1)} F^{(1) i j}\right)+\frac{\sqrt{2}}{4 g \tilde{v}^{2}} \operatorname{Tr}\left[\epsilon^{i j} F_{i j}^{(1)} \tilde{\psi}_{I \alpha}\left(\tilde{\psi}^{I \dagger} \gamma_{3}\right)^{\alpha}\right]+\frac{1}{4 \tilde{v}^{2}} \operatorname{Tr}\left[\tilde{\psi}_{I \alpha}\left(\tilde{\psi}^{\dagger I} \gamma_{3}\right)^{\alpha}\right]^{2}\right]$.

The first term in the above looks like a 2d YM kinetic term, with coupling

$$
g_{Y M} \equiv g \tilde{v}=\frac{2 \pi \sqrt{2} \tilde{v}}{k R} .
$$

Note that half of the gauge fields were fixed by the VEV. Moreover, if one chose to keep the scalar field fluctuations the second gauge field would also have to appear in a 'kinetic term', multiplied by said fluctuations. In that event it is best to think of the latter as an interaction term, with the constraints fixing both $\phi_{1}$ and $\phi_{2}$ but the second gauge field remaining unfixed.

In the mass-deformed case, one keeps a kinetic term involving both gauge fields. This fixes both $\phi_{1}$ and $\phi_{2}$ even when expanding the theory around the fuzzy sphere vacuum and keeping only leading terms by setting their fluctuations to zero.

\subsubsection{Higgsed action at large $\tilde{v}$ and D1-brane in 9d}

We just saw that by eliminating the $\phi_{i}$ 's in the absence of scalar fields one gets a nontrivial action with a $2 \mathrm{~d}$ YM kinetic term. This renders it in principle compatible with a D1-brane 
interpretation. In the following we would like to show that at large $\tilde{v}$ and large $k$, as imposed by the finiteness of (2.20) with $\frac{\tilde{v}}{k}=$ fixed, we will obtain a D1-brane low-energy theory in $9 \mathrm{~d}$ flat space.

We begin by defining

$$
\begin{aligned}
\tilde{B}_{y}=\phi_{1}-\phi_{2}, & \tilde{Q}_{y}=\phi_{1}+\phi_{2} \\
B_{i}=\frac{1}{2}\left(A_{i}^{(1)}-A_{i}^{(2)}\right), & Q_{i}=\frac{1}{2}\left(A_{i}^{(1)}+A_{i}^{(2)}\right) \\
F_{i j}^{B}=\frac{1}{2}\left(F_{i j}^{(1)}-F_{i j}^{(2)}\right), & F_{i j}=\frac{1}{2}\left(F_{i j}^{(1)}+F_{i j}^{(2)}\right),
\end{aligned}
$$

which implies

$$
\begin{aligned}
F_{i j} & =\partial_{i} Q_{j}-\partial_{j} Q_{i}+i\left[Q_{i}, Q_{j}\right]+i\left[B_{i}, B_{j}\right] \\
F_{i j}^{B} & =\tilde{D}_{i} B_{j}-\tilde{D}_{j} B_{i} \\
\tilde{D}_{i} & \equiv \partial_{i}+i\left[Q_{i}, \cdot\right] .
\end{aligned}
$$

The $\phi_{i}$-dependent terms in the action (2.6) can then be rewritten as

$$
\begin{aligned}
S_{\phi}=\int d^{2} x\left[\frac{k}{4 \pi} \epsilon^{i j} \operatorname{Tr}(\right. & \left.\tilde{B}_{y} F_{i j}+\tilde{Q}_{y} F_{i j}^{B}\right)+\frac{1}{2 R} \operatorname{Tr}\left(\tilde{\psi}^{I \dagger} \gamma_{3}\left(\left[\tilde{\psi}_{I}, \tilde{Q}_{y}\right]-\left\{\tilde{\psi}_{I}, \tilde{B}_{y}\right\}\right)\right) \\
+ & \left.\frac{1}{4 R^{2}} \operatorname{Tr}\left(\left[\tilde{C}^{I}, \tilde{Q}_{y}\right]-\left\{\tilde{C}^{I}, \tilde{B}_{y}\right\}\right)\left(\left[\tilde{C}_{I}^{\dagger}, \tilde{Q}_{y}\right]+\left\{\tilde{C}_{I}^{\dagger}, \tilde{B}_{y}\right\}\right)\right] .
\end{aligned}
$$

We expand the scalars around the VEV solution as

$$
\begin{array}{rr}
\tilde{C}^{1}=\tilde{v}+\rho+i \sigma, & \rho=\rho_{0}+i \rho_{a} T^{a} \\
\tilde{C}^{I^{\prime}}=X^{I^{\prime}}+i X^{I^{\prime}+4}, & \sigma=\sigma_{0}+i \sigma_{a} T^{a} \\
X^{A^{\prime \prime}}=X_{0}^{A^{\prime \prime}}+i X_{a}^{A^{\prime \prime}} T^{a}, &
\end{array}
$$

where $T^{a}$ are $\mathrm{SU}(N)$ generators, $I^{\prime}=2,3,4, A^{\prime \prime}=\left(I^{\prime}, I^{\prime}+4\right)$ and the subscript 0 indicates the trace part.

The $\phi_{i}$-dependent action (2.23) becomes to leading order in $\tilde{v}$

$$
\begin{array}{r}
S_{\phi}=\int d^{2} x\left[\frac{k}{4 \pi} \epsilon^{i j} \operatorname{Tr}\left(\tilde{B}_{y} F_{i j}+\tilde{Q}_{y} F_{i j}^{B}\right)+\frac{1}{2 R} \operatorname{Tr}\left(\tilde{\psi}^{I \dagger} \gamma_{3}\left(\left[\tilde{\psi}_{I}, \tilde{Q}_{y}\right]-\left\{\tilde{\psi}_{I}, \tilde{B}_{y}\right\}\right)\right)\right. \\
\left.-\frac{\tilde{v}^{2}}{R^{2}} \operatorname{Tr}\left(\tilde{B}_{y}^{2}\right)+\mathcal{O}(\tilde{v})\right],
\end{array}
$$

i.e. remains independent of the scalars.

In exact analogy to the $3 \mathrm{~d}$ case $[4,15],{ }^{3}$ we obtain for the scalar potential

$$
\frac{4 \pi^{2}}{3 k^{2} R^{2}} V_{6}\left(\tilde{C}^{I}\right)=-\frac{4 \pi^{2} \tilde{v}^{2}}{k^{2} R^{2}} \operatorname{Tr}\left[X^{A^{\prime}}, X^{B^{\prime}}\right]^{2}+\mathcal{O}(\tilde{v})=\frac{g_{Y M}^{2}}{2} \operatorname{Tr}\left[X^{A^{\prime}}, X^{B^{\prime}}\right]^{2}\left(1+\mathcal{O}\left(\frac{1}{\tilde{v}}\right)\right)
$$

where $A^{\prime}=2, \ldots, 8$ and $X^{5} \equiv \sigma$. For the fermionic potential we have

$$
\begin{array}{r}
\frac{i g \tilde{v}}{\sqrt{2}} V_{f e r m}=-\frac{i g_{Y M}}{\sqrt{2}} \operatorname{Tr}[ \\
2 \rho\left(\tilde{\psi}^{\dagger J} \tilde{\psi}_{J}-\tilde{\psi}_{J} \tilde{\psi}^{\dagger J}\right)-2 \tilde{\psi}_{1}\left(\tilde{C}_{I}^{\dagger} \tilde{\psi}^{\dagger I}-\tilde{\psi}^{\dagger I} \tilde{C}_{I}^{\dagger}\right)+2 \tilde{\psi}^{\dagger 1}\left(\tilde{C}^{I} \tilde{\psi}_{I}-\tilde{\psi}_{I} \tilde{C}^{I}\right) \\
\left.+2 \epsilon^{I^{\prime} J^{\prime} K^{\prime}} \tilde{\psi}_{I^{\prime}} \tilde{C}_{J^{\prime}}^{\dagger} \tilde{\psi}_{K^{\prime}}-2 \epsilon_{I^{\prime} J^{\prime} K^{\prime}} \tilde{\psi}^{\dagger I^{\prime}} \tilde{C}^{J^{\prime}} \tilde{\psi}^{\dagger K^{\prime}}\right]\left(1+\mathcal{O}\left(\frac{1}{\tilde{v}}\right)\right) \quad(2.27)
\end{array}
$$

\footnotetext{
${ }^{3}$ The calculation is identical and we will hence omit it at this stage. See also [16].
} 
and one similarly obtains the anticipated SYM Yukawa term

$$
-\frac{1}{2} f^{a b c} X_{a}^{A^{\prime}} \bar{\psi}_{b} \Gamma^{A^{\prime}} \psi_{c}+\mathcal{O}\left(\frac{1}{\tilde{v}}\right)
$$

by rearranging the fermions into $\mathrm{SO}(1,1) \times \mathrm{SO}(7)$ spinors, again in direct analogy with [15]. Note that the scalar $\rho_{a}$ (the real part of $\tilde{C}^{1}$ ) does not appear in either the final bosonic or fermionic potentials, as was also the case in $3 \mathrm{~d}$.

We now move on to eliminate the auxiliary fields $\tilde{B}_{y}$ and $\tilde{Q}_{y}$ from the action $S_{\phi}$. Varying (2.25) with respect to $\tilde{B}_{y}$ and $\tilde{Q}_{y}$, we obtain

$$
\begin{aligned}
\tilde{B}_{y} & =\frac{\sqrt{2} R}{4 g \tilde{v}^{2}} \epsilon^{i j} F_{i j}-\frac{R}{4 \tilde{v}^{2}}\left(\tilde{\psi}_{I \alpha}\left(\tilde{\psi}^{I \dagger} \gamma_{3}\right)^{\alpha}+\tilde{\psi}^{I \dagger} \gamma_{3} \tilde{\psi}_{I}\right) \\
\epsilon^{i j} F_{i j}^{B} & =\frac{2 \pi}{k R}\left(\tilde{\psi}_{I \alpha}\left(\tilde{\psi}^{I \dagger} \gamma_{3}\right)^{\alpha}+\tilde{\psi}^{I \dagger} \gamma_{3} \tilde{\psi}_{I}\right)
\end{aligned}
$$

and substituting back we find

$$
\begin{aligned}
S_{\phi}= & \int d^{2} x\left[-\frac{1}{4 g_{Y M}^{2}} F_{i j} F^{i j}-\frac{\sqrt{2}}{8 g \tilde{v}^{2}} \epsilon^{i j} F_{i j}\left(\tilde{\psi}_{\alpha}^{I}\left(\tilde{\psi}^{I \dagger} \gamma_{3}\right)^{\alpha}+\tilde{\psi}^{I \dagger} \gamma_{3} \tilde{\psi}_{I}\right)\right. \\
& \left.-\frac{1}{16 \tilde{v}^{2}}\left(\tilde{\psi}_{\alpha}^{I}\left(\tilde{\psi}^{I \dagger} \gamma_{3}\right)^{\alpha}+\tilde{\psi}^{I \dagger} \gamma_{3} \tilde{\psi}_{I}\right)^{2}\right]\left(1+\mathcal{O}\left(\frac{1}{\tilde{v}}\right)\right) \\
\rightarrow & -\frac{1}{4 g_{Y M}^{2}} \int d^{2} x F_{i j} F^{i j}+\mathcal{O}\left(\frac{1}{\tilde{v}}\right),
\end{aligned}
$$

that is only the $F_{i j}$ YM kinetic term survives in the large $\tilde{v}$ limit. We note that in the above there is no YM kinetic term for $F_{i j}^{B}$ and that to $\mathcal{O}(1)$ in $S_{\phi}$, the $\tilde{Q}_{y}$ field drops out from the action, despite $\tilde{Q}_{y}$ not being fixed to leading order.

Apart from the $\phi_{i}$-dependent part of the action, one also needs to take into consideration the $\tilde{v}^{2} B_{i} B^{i}$ mass-terms coming from the covariant kinetic term for the scalars, $\left|D_{i} \tilde{C}^{I}\right|^{2}$. Even though $B_{i}$ is an adjoint field under $Q_{i}$, it is itself the gauge field for a shift symmetry that acts as

$$
\begin{aligned}
& B_{i} \rightarrow B_{i}-\tilde{D}_{i} \lambda \\
& W \rightarrow W+\alpha \lambda,
\end{aligned}
$$

with $W=\sigma_{0}+i \rho_{a} T^{a}[16]$ and $\alpha$ an appropriate combination of the parameters of the theory. In order to proceed we observe that in the large- $k$ limit the $\tilde{Q}_{y}$ constraint becomes $\epsilon^{i j} F_{i j}^{B}=0$, which for topologically trivial fields is solved by a pure gauge condition

$$
B_{i}=\tilde{D}_{i} \lambda .
$$

One might be tempted to think of this as a trivial solution but this is not the case. In fact, this is just a signal of the ordinary Higgs mechanism where the gauge field 'eats' the Goldstone boson to become massive. Indeed, in $2 \mathrm{~d}$ the $\mathrm{YM}$ gauge field is nondynamical, while a massive (Proca) vector field with Lagrangian density

$$
-\frac{1}{4 g_{Y M}^{2}} F_{i j}^{2}-m^{2} A_{i}^{2}
$$


has one dynamical degree of freedom. For the case at hand there is no YM kinetic term but we still have a mass for $B_{i}$ from the scalar kinetic term, which renders it dynamical. In the final step, we substitute the single dynamical mode of $B_{i}$ through (2.32), in effect replacing the Goldstone mode by $\lambda$.

At this point we should note that by substituting (2.32) in $F_{i j}^{B}$ one gets

$$
F_{i j}^{B}=\left[\partial_{i} Q_{j}-\partial_{j} Q_{i}+i\left[Q_{i}, Q_{j}\right], \lambda\right]=\left[F_{i j}-i\left[B_{i}, B_{j}\right], \lambda\right]
$$

instead of zero. But, as we will see below eq. (2.37), $\lambda$ and $B_{i}$ are of order $\mathcal{O}\left(\frac{1}{\tilde{v}}\right)$ because Higgsing implies a term $\tilde{v}^{2} B_{i} B^{i}$. This in turn means that $F_{i j}^{B}$ is automatically of order $\mathcal{O}\left(\frac{1}{\tilde{v}}\right)$ as required by $(2.29)$, so imposing $(2.32)$ would at first seem redundant. Yet for a purely bosonic background $F_{i j}^{B}$ is zero to better than $\mathcal{O}\left(\frac{1}{\tilde{v}}\right)$ accuracy and $(2.32)$ is needed. Since $B_{i} \sim \mathcal{O}\left(\frac{1}{\tilde{v}}\right) \rightarrow 0$, we have from $(2.22)$

$$
F_{i j} \simeq \partial_{i} Q_{j}-\partial_{j} Q_{i}+i\left[Q_{i}, Q_{j}\right]
$$

as required for a Yang-Mills theory. Then we want

$$
\left|\left[F_{i j}, \lambda\right]\right| \ll|\lambda|
$$

which can be achieved in two different ways: Firstly through a restriction on the fields, by $F_{i j}$ and $\lambda$ or $Q_{i}$ and $B_{i}$ belonging to commuting subgroups of $\mathrm{SU}(N)$. Secondly, we can consider that the $\mathrm{U}(1)$ (commuting) component of $\lambda, \lambda_{0}$, is much larger than the $\mathrm{SU}(N)$ components $\lambda_{a}$. This latter possibility has a nice physical interpretation, as we shall see.

Returning to the calculation, the covariant derivative on a scalar $\tilde{C}$ becomes in terms of $\lambda$

$$
D_{i} \tilde{C}=\partial_{i} \tilde{C}+i\left[Q_{i}, \tilde{C}\right]+i\left\{B_{i}, \tilde{C}\right\}=\partial_{i} \tilde{C}+i\left[Q_{i}, \tilde{C}\right]+i\left\{D_{i} \lambda, \tilde{C}\right\} \equiv \tilde{D}_{i} \tilde{C}+i\left\{\partial_{i} \lambda, \tilde{C}\right\}
$$

with the same action for the derivative on the fermions.

Now consider the $\mathrm{SU}(N) \subset \mathrm{U}(N)$ part. We define $\tilde{\lambda} \equiv \tilde{v} \lambda=\tilde{\lambda}_{a} T^{a}$, obtaining in the large $\tilde{v}$ limit

$$
\begin{aligned}
& \left|D_{i} \tilde{C}^{I^{\prime}}\right|^{2} \rightarrow\left|\tilde{D}_{i} \tilde{C}^{I^{\prime}}\right|^{2} \\
& \operatorname{Tr}\left|D_{i} \tilde{C}^{1}\right|^{2} \rightarrow \operatorname{Tr}\left|D_{i} \sigma\right|^{2}+\left(\partial_{i} \rho_{0}\right)^{2}+\left|D_{i}\left(\rho_{a}+2 \tilde{\lambda}_{a}\right)\right|^{2},
\end{aligned}
$$

while the fermionic kinetic term becomes just

$$
-i \operatorname{Tr}\left(\tilde{\psi}^{I \dagger} \gamma^{i} D_{i} \tilde{\psi}_{I}\right) \rightarrow-i \operatorname{Tr}\left(\tilde{\psi}^{I \dagger} \gamma^{i} \tilde{D}_{i} \tilde{\psi}_{I}\right)
$$

Therefore as in the usual Higgs mechanism $D_{i} \tilde{\lambda}_{a}$ comes only in combination with $D_{i} \rho_{a}$, where $\rho_{a}$ is the Goldstone boson that does not appear in the scalar potential. This is how $\lambda_{a}$ replaces the original nonabelian Goldstone boson.

For the part of $\lambda$ which is in the $\mathrm{U}(1)$ centre of $\mathrm{U}(N), \lambda_{0}$, the kinetic term for the scalars becomes

$$
\left|D_{i} \tilde{C}^{I}\right|^{2}=\left|\tilde{D}_{i}\left(\tilde{C}^{I} e^{2 i \lambda_{0}}\right)\right|^{2}
$$


Upon taking $k$ and $\tilde{v}$ large the theory undergoes an effective compactification, according to the orbifold picture of [5]. This corresponds to the vanishing of a scalar trace degree of freedom, which in this case is $\sigma_{0} \cdot{ }^{4}$ The identification $\tilde{C}^{I} \sim e^{-2 i \lambda_{0}} \tilde{C}^{I}$ signals that this degree of freedom is now carried by $\lambda_{0}$. Hence we also see that when solving the restriction (2.36) by $\lambda_{0} \gg \lambda_{a}$, the interpretation is that the relative separations in the compactified direction, $\lambda_{a}$, are much smaller than the center of mass position $\lambda_{0}$.

Putting everything together, the final action is the action of a D1-brane in 9d flat space, with 7 nontrivial transverse scalars and one Goldstone boson $(\lambda)$, encoding information about the 10th (compact) dimension.

\subsubsection{Higgsed action at large $\tilde{v}$ and D1-brane in 10d}

In getting the D1 action in $9 \mathrm{~d}$ we eliminated the auxiliary scalars $\phi_{i}$, or equivalently $\tilde{B}_{y}$ and $\tilde{Q}_{y}$, via their equations of motion. However, one can easily observe that upon performing a partial integration $B_{i}$ also appears as an auxiliary field in the dimensionally reduced action (2.6). In this section we will examine the consequences of eliminating $\tilde{B}_{y}$ and $B_{i}$ from the action instead of $\phi_{i}$.

We begin with the large- $\tilde{v}$-limit expression coming from (2.23) plus the mass term for $B_{i}$

$$
S_{B_{i}}=\int d^{2} x\left[\frac{k}{4 \pi} \epsilon^{i j} \tilde{Q}_{y} F_{i j}^{B}-4 \tilde{v}^{2} B_{i} B^{i}+\mathcal{O}(\tilde{v})\right] .
$$

Solving for the $\tilde{B}_{y}$ and $B_{i}$ auxiliary fields, we obtain

$$
\begin{aligned}
\tilde{B}_{y} & =\frac{\sqrt{2} R}{4 g \tilde{v}^{2}} \epsilon^{i j} F_{i j}+\frac{R}{4 \tilde{v}^{2}}\left(\tilde{\psi}_{I \alpha}\left(\tilde{\psi}^{I \dagger} \gamma_{3}\right)^{\alpha}-\tilde{\psi}^{I \dagger} \gamma_{3} \tilde{\psi}_{I}\right) \\
B_{i} & =\frac{k}{16 \pi \tilde{v}^{2}} \epsilon_{i j} D^{j} \tilde{Q}_{y} .
\end{aligned}
$$

Substituting back in the action $S_{\phi}+S_{B_{i}}$, we arrive at

$$
\begin{aligned}
S_{a u x}= & \int d^{2} x\left[-\frac{1}{4 g_{Y M}^{2}} F_{i j} F^{i j}+\frac{\sqrt{2}}{8 g \tilde{v}^{2}} \epsilon^{i j} F_{i j}\left(\tilde{\psi}^{I \alpha}\left(\tilde{\psi}^{I \dagger} \gamma_{3}\right)^{\alpha}-\tilde{\psi}^{I \dagger} \gamma_{3} \tilde{\psi}_{I}\right)\right. \\
& \left.+\frac{1}{16 \tilde{v}^{2}}\left(\tilde{\psi}^{I \alpha}\left(\tilde{\psi}^{I \dagger} \gamma_{3}\right)^{\alpha}-\tilde{\psi}^{I \dagger} \gamma_{3} \tilde{\psi}_{I}\right)^{2}-\frac{k^{2}}{(8 \pi \tilde{v})^{2}}\left(D_{i} \tilde{Q}_{y}\right)^{2}\right]+\mathcal{O}\left(\frac{1}{\tilde{v}}\right) \\
\rightarrow & \int d^{2} x\left[-\frac{1}{4 g_{Y M}^{2}} F_{i j} F^{i j}-\frac{1}{8 R^{2} g_{Y M}^{2}}\left(D_{i} \tilde{Q}_{y}\right)^{2}\right]+\mathcal{O}\left(\frac{1}{\tilde{v}}\right)
\end{aligned}
$$

We note from (2.42) that $B_{i}$ is of order $\mathcal{O}\left(\frac{1}{\tilde{v}}\right)$, as also mentioned in the previous subsection. Hence, $F_{i j}$ in (2.22) reduces to the usual Yang-Mills form (2.35). One already sees that $\tilde{Q}_{y}$ plays the role of an extra dynamical scalar similar to $\lambda$ from the previous analysis, although unlike that case it will combine with the others to make the scalar potential of the D1-brane in 10d. For that, we are missing the terms $g_{Y M}^{2}\left[\tilde{X}^{1}, \tilde{X}^{A^{\prime}}\right]^{2}$.

\footnotetext{
${ }^{4}$ For a precise treatment of the U(1) factors in the Higgsing of the ABJM theory see [16].
} 
Indeed, in the second line of (2.23) one has a term

$$
\frac{1}{4 R^{2}}\left[\tilde{C}^{I}, \tilde{Q}_{y}\right]\left[\tilde{C}_{I}^{\dagger}, \tilde{Q}_{y}\right]
$$

which gives the missing terms upon the identification $\tilde{X}_{a}^{1}=\frac{1}{2 R g_{Y M}} \tilde{Q}_{y a}$. The latter also gives the correct scalar field normalisation in (2.43). Similarly, there is a missing term in the fermionic potential which is provided by the term

$$
\frac{1}{2 R} \tilde{\psi}^{I \dagger} \gamma_{3}\left[\tilde{\psi}_{I}, \tilde{Q}_{y}\right]
$$

in $(2.23)$.

All in all, in the large $\tilde{v}$ limit and in terms of $\tilde{Q}_{y}, Q_{i}$, we obtain the D1-brane action in 10d flat space.

\subsubsection{Higgsed action and T-duality}

The fact that we can obtain the low-energy D1-brane action in $9 \mathrm{~d}$ or $10 \mathrm{~d}$, depending on what auxiliary fields we choose to eliminate from the 'master' action (2.6), might seem strange at first. However, we will see shortly that one can switch between them using the Buscher rules [17], i.e. a field theoretic version of T-duality.

We should clarify that in our setup the M-theory direction has already been compactified on a circle of radius $R$. That led to a worldvolume reduction of the M2-brane theory. On the other hand, the T-duality we are referring to here in the $\tilde{v} \rightarrow \infty$ limit is on an additional compact dimension of radius $R_{10}$, which is transverse to the branes and hence involves a worldvolume scalar field. As a result, one of the 10 dimensions of string theory is compactified on a very small/very large radius.

We start with a brief review of the rules for a string worldsheet in some nontrivial background. Taking

$$
S=\int d^{2} \sigma \sqrt{\gamma} \gamma^{\mu \nu} g_{a b} \partial_{\mu} x^{a} \partial_{\nu} x^{b}
$$

one writes it in a first order form as

$$
S=\int d^{2} \sigma\left[\sqrt{\gamma} \gamma^{\mu \nu}\left(g_{00} V_{\mu} V_{\nu}+2 g_{0 i} V_{\mu} \partial_{\nu} x^{i}+g_{i j} \partial_{\mu} x^{i} \partial_{\nu} x^{j}\right)+2 \epsilon^{\mu \nu} \hat{x}^{0} \partial_{\mu} V_{\nu}\right] .
$$

Then by varying with respect to $\hat{x}^{0}$ we get $\epsilon^{\mu \nu} \partial_{\mu} V_{\nu}=0$, solved by $V_{\mu}=\partial_{\mu} x^{0}$, which when plugged in (2.47) gives back (2.46). If we instead solve for $V_{\mu}=\frac{\hat{g}_{00}}{\sqrt{\gamma}} \epsilon_{\nu \mu} \partial^{\nu} \hat{x}^{0}-\hat{g}_{0 i} \partial_{\mu} \hat{x}^{i}$ and substitute back into the action, we get the T-dual expression

$$
S=\int d^{2} \sigma\left[\sqrt{\gamma} \gamma^{\mu \nu} \hat{g}_{a b} \partial_{\mu} \hat{x}^{a} \partial_{\nu} \hat{x}^{b}+\epsilon^{\mu \nu} \hat{h}_{a b} \partial_{\mu} \hat{x}^{a} \partial_{\nu} \hat{x}^{b}\right]
$$

where the background fields are the T-dual ones:

$$
\hat{g}_{00}=\frac{1}{g_{00}} ; \quad \hat{g}_{i j}=g_{i j}-\frac{g_{0 i} g_{0 j}}{g_{00}} ; \quad \hat{h}_{0 i}=\frac{g_{0 i}}{g_{00}}
$$


with the remaining components of $\hat{h}_{a b}$ zero and $\hat{x}^{i}=x^{i}$.

In order to compare the above with our case, we concentrate on the relevant terms in the two first order 'master' actions ${ }^{5}$

$$
\begin{aligned}
S_{D 1} & =-\int\left[4 \tilde{v}^{2} B_{i}^{2}-\frac{k}{4 \pi} \epsilon^{i j} \tilde{Q}_{y} F_{i j}^{B}\right] \\
S_{\text {Buscher }} & =\int\left[g_{00} V_{\mu} V^{\mu}+2 \epsilon^{\mu \nu} \hat{x}^{0} \partial_{\mu} V_{\nu}\right],
\end{aligned}
$$

which leads to the identifications

$$
\begin{aligned}
V_{\mu} & \leftrightarrow B_{i} \\
g_{00} & \leftrightarrow 4 \tilde{v}^{2} \\
\hat{x}^{0} & \leftrightarrow-\frac{k}{4 \pi} \tilde{Q}_{y} \\
x^{0} & \leftrightarrow \lambda .
\end{aligned}
$$

The T-dual second order forms are compared in a similar manner:

$$
\begin{aligned}
g_{00} \partial_{\mu} x^{0} \partial^{\mu} x^{0} & \leftrightarrow 4 \tilde{v}^{2}\left(\partial_{i} \lambda\right)^{2} \\
\frac{1}{g_{00}} \partial_{\mu} \hat{x}^{0} \partial^{\mu} \hat{x}^{0} & \leftrightarrow \frac{1}{4 \tilde{v}^{2}}\left[D_{i}\left(-\frac{k \tilde{Q}_{y}}{4 \pi}\right)\right]^{2}
\end{aligned}
$$

We therefore see that the $R_{10} \rightarrow \frac{1}{R_{10}}$ T-duality in our case becomes $2 \tilde{v} \rightarrow \frac{1}{2 \tilde{v}}$. This indicates that while in the first formulation

$$
\lambda \sim \frac{1}{\tilde{v}}
$$

i.e. $\lambda$ takes values on a circle of small radius $\propto \frac{1}{\tilde{v}}$, as expected for a D1-brane in $9 \mathrm{~d}$, in the T-dual formulation

$$
\frac{k \tilde{Q}_{y}}{4 \pi} \sim \tilde{v}
$$

and the T-dual field takes values on a circle of large radius $\propto \tilde{v}$, with the corresponding direction decompactified as expected for a D1-brane in 10d.

One is perhaps more familiar with T-duality exchanging the momentum $p=\frac{n}{R_{10}}$ with winding $w=\frac{m R_{10}}{\alpha^{\prime}}$, or $n$ with $m$ in the expansion of the physical compact direction $R_{10} x^{0}=$ $X^{0}=\frac{n \alpha^{\prime}}{R_{10}} \tau+m R_{10} \sigma+\ldots$. But as is well-known, in the Buscher form of T-duality this arises because the two dual coordinates $x^{0}$ and $\hat{x}^{0}$ are solutions to the same master $V_{\mu}$ as

$$
V_{\mu}=\partial_{\mu} x_{0}=\frac{\hat{g}_{00}}{\sqrt{\gamma}} \epsilon_{\nu \mu} \partial^{\nu} \hat{x}^{0}-\hat{g}_{0 i} \partial_{\mu} \hat{x}^{i} .
$$

Taking the particular case $g_{0 i}=0$ and the conformal gauge $\gamma_{\mu \nu}=\delta_{\mu \nu}$, one gets for the physical coordinates $\partial_{\mu}\left(R_{10} x^{0}\right)=\epsilon_{\mu \nu} \partial^{\nu}\left(\frac{\alpha^{\prime}}{R_{10}} \hat{x}^{0}\right)$, or $\partial_{\tau}\left(X^{0}\right)=\partial_{\sigma}\left(X^{0^{\prime}}\right), \partial_{\sigma}\left(X^{0}\right)=\partial_{\tau}\left(X^{0^{\prime}}\right)$, i.e. exactly exchanging $p$ with $w$. In our case, we can use the map (2.51) to obtain the exact same momentum $\leftrightarrow$ winding exchange in the limit where we only keep the compact abelian scalar from the action. Of course, in the full theory it is not clear how the T-duality acts on full states.

\footnotetext{
${ }^{5}$ The difference in the overall sign is due to different conventions.
} 


\subsection{The $N=1$ case}

We next turn to the study of the abelian case, which we will further interpret in the following section. By setting $N=1$ in the ABJM action, one obtains a theory for a single supermembrane on $\mathbb{C}^{4} / \mathbb{Z}_{k}[1,18]$

$$
S_{\mathrm{ABJM}}^{N=1}=\int d^{3} x\left[\frac{k}{4 \pi} \epsilon^{\mu \nu \lambda}\left(A_{\mu}^{(1)} \partial_{\nu} A_{\lambda}^{(1)}-A_{\mu}^{(2)} \partial_{\nu} A_{\lambda}^{(2)}\right)-i \psi^{I \dagger} \gamma^{\mu} D_{\mu} \psi_{I}-D_{\mu} C_{I}^{\dagger} D^{\mu} C^{I}\right],
$$

where

$$
D_{\mu} C^{I}=\partial_{\mu} C^{I}-i\left(A_{\mu}^{(1)}-A_{\mu}^{(2)}\right) C^{I} .
$$

By applying the same dimensional reduction procedure as for the general nonabelian case we obtain the $2 \mathrm{~d}$ action

$$
\begin{aligned}
& S= \int d^{2} x\left[-D_{i} \tilde{C}_{I}^{\dagger} D^{i} \tilde{C}^{I}-i \tilde{\psi}^{I \dagger} \gamma^{i} D_{i} \tilde{\psi}_{I}\right]+S_{\phi} \\
& S_{\phi}=\int d^{2} x\left[\frac{\sqrt{2}}{2 g R} \epsilon^{i j}\left(\phi_{1} F_{i j}^{(1)}-\phi_{2} F_{i j}^{(2)}\right)-R^{-2}\left(\tilde{C}^{I} \tilde{C}_{I}^{\dagger}\right)\left(\phi_{1}-\phi_{2}\right)^{2}\right. \\
&\left.-R^{-1}\left(\phi_{1}-\phi_{2}\right) \tilde{\psi}^{I \dagger} \gamma_{3} \psi_{I}\right] .
\end{aligned}
$$

Then varying with respect to $\phi_{1}, \phi_{2}$ we get the constraints

$$
\begin{aligned}
\phi_{1}-\phi_{2} & =\frac{R \sqrt{2}}{4 g} \frac{\epsilon^{i j} F_{i j}^{(1)}}{\tilde{C}^{I} \tilde{C}_{I}^{\dagger}}-\frac{R}{2 \tilde{C}^{I} \tilde{C}_{I}^{\dagger}}\left(\tilde{\psi}^{I \dagger} \gamma_{3} \psi_{I}\right) \\
\epsilon^{i j} F_{i j}^{(2)} & =\epsilon^{i j} F_{i j}^{(1)}
\end{aligned}
$$

and once again $\phi_{1}-\phi_{2}$ as well as $F_{i j}^{(2)}$ can be solved in terms of other fields. Substituting back in $S_{\phi}$ :

$$
\begin{aligned}
S_{\phi} & =\int d^{2} x\left[-\frac{1}{4 g^{2} \tilde{C}^{I} \tilde{C}_{I}^{\dagger}}\left(F_{i j}^{(1)}\right)^{2}+\frac{1}{4 \tilde{C}^{I} \tilde{C}_{I}^{\dagger}}\left(\tilde{\psi}^{I \dagger} \gamma_{3} \tilde{\psi}_{I}\right)^{2}-\frac{\sqrt{2}}{4 g \tilde{C}^{I} \tilde{C}_{I}^{\dagger}} \epsilon^{i j} F_{i j}^{(1)} \tilde{\psi}^{I \dagger} \gamma_{3} \tilde{\psi}\right] \\
& =\int d^{2} x \frac{\tilde{C}^{I} \tilde{C}_{I}^{\dagger}}{R^{2}}\left[\frac{R \sqrt{2}}{4 g} \frac{\epsilon^{i j} F_{i j}^{(1)}}{\tilde{C}^{I} \tilde{C}_{I}^{\dagger}}-\frac{R}{2 \tilde{C}^{I} \tilde{C}_{I}^{\dagger}}\left(\tilde{\psi}^{I \dagger} \gamma_{3} \psi_{I}\right)\right]^{2}
\end{aligned}
$$

The equation of motion for $A_{i}^{(1)}$ gives

$$
\partial_{i}\left[\frac{R \sqrt{2}}{4 g} \frac{\epsilon^{i j} F_{i j}^{(1)}}{\tilde{C}^{I} \tilde{C}_{I}^{\dagger}}-\frac{R}{2 \tilde{C}^{I} \tilde{C}_{I}^{\dagger}}\left(\tilde{\psi}^{I \dagger} \gamma_{3} \psi_{I}\right)\right]=0
$$

i.e. the bracket is a constant, with $S_{\phi}$ proportional to the square of this bracket. Note that due to the constraints $(2.60)$ we can choose a gauge in which $A_{i}^{(1)}=A_{i}^{(2)}$, such that the covariant derivative reduces to the partial derivative. 


\subsection{Supersymmetry}

The action has 12 supersymmetries for any value of $N$, found by dimensional reduction of the ABJM supersymmetries. The supersymmetry rules in $3 \mathrm{~d}$ are

$$
\begin{aligned}
\delta C^{I} & =i \omega^{I J} \psi_{J} \\
\delta C_{I}^{\dagger} & =i \psi^{\dagger J} \omega_{I J} \\
\delta \psi_{I} & =-\gamma_{\mu} \omega_{I J} D_{\mu} C^{J}+\frac{2 \pi}{k}\left(-\omega_{I J}\left(C^{K} C_{K}^{\dagger} C^{J}-C^{J} C_{K}^{\dagger} C^{K}\right)+2 \omega_{K L} C^{K} C_{I}^{\dagger} C^{L}\right) \\
\delta \psi^{I \dagger} & =D_{\mu} C_{J}^{\dagger} \gamma_{\mu} \omega^{I J}+\frac{2 \pi}{k}\left(-\left(C_{J}^{\dagger} C^{K} C_{K}^{\dagger}-C_{K}^{\dagger} C^{K} C_{J}^{\dagger}\right)+2 C_{L}^{\dagger} C^{I} C_{K}^{\dagger} \omega^{K L}\right) \\
\delta A_{\mu}^{(1)} & =-\frac{\pi}{k}\left(C^{I} \psi^{J \dagger} \gamma_{\mu} \omega^{I J}+\omega^{I J} \gamma_{\mu} \psi_{I} C_{J}^{\dagger}\right) \\
\delta A_{\mu}^{(2)} & =\frac{\pi}{k}\left(\psi^{I \dagger} C^{J} \gamma_{\mu} \omega_{I J}+\omega^{I J} \gamma_{\mu} C_{I}^{\dagger} \psi_{j}\right)
\end{aligned}
$$

Dimensionally reducing, we obtain

$$
\begin{aligned}
\delta \tilde{C}^{I=} & i \omega^{I J} \tilde{\psi}_{J} \\
\delta \tilde{\psi}_{I}= & -\gamma_{i} \omega_{I J} D_{i} \tilde{C}^{J}-\frac{i}{R} \gamma_{3} \omega_{I J}\left(\tilde{C}^{J} \phi_{2}-\phi_{1} \tilde{C}^{J}\right) \\
& +\frac{g}{\sqrt{2}}\left[-\omega_{I J}\left(\tilde{C}^{K} \tilde{C}_{K}^{\dagger} \tilde{C}^{J}-\tilde{C}^{J} \tilde{C}_{K}^{\dagger} \tilde{C}^{K}\right)+2 \omega_{K L} \tilde{C}^{K} \tilde{C}_{I}^{\dagger} \tilde{C}^{L}\right] \\
\delta A_{i}^{(1)}= & -\frac{g}{2 \sqrt{2}}\left(\tilde{C}^{I} \tilde{\psi}^{J \dagger} \gamma_{i} \omega_{I J}+\omega^{I J} \gamma_{i} \tilde{\psi}_{I} \tilde{C}_{J}^{\dagger}\right) \\
\delta A_{i}^{(2)=} & +\frac{g}{2 \sqrt{2}}\left(\tilde{\psi}^{I} \tilde{C}^{J} \gamma_{i} \omega_{I J}+\omega^{I J} \gamma_{i} \tilde{C}_{I} \tilde{\psi}_{J}\right) \\
\delta \phi_{1}= & -\frac{R g}{2 \sqrt{2}}\left(\tilde{C}^{I} \tilde{\psi}^{J \dagger} \gamma_{3} \omega_{I J}+\omega^{I J} \gamma_{3} \tilde{\psi}_{I} \tilde{C}_{J}^{\dagger}\right) \\
\delta \phi_{2}= & +\frac{R g}{2 \sqrt{2}}\left(\tilde{\psi}^{I} \tilde{C}^{J} \gamma_{3} \omega_{I J}+\omega^{I J} \gamma_{3} \tilde{C}_{I} \tilde{\psi}_{J}\right) .
\end{aligned}
$$

Restricting to $N=1$

$$
\begin{aligned}
\delta \tilde{C}^{I} & =i \omega^{I J} \tilde{\psi}_{J} \\
\delta \tilde{\psi}_{I} & =-\frac{1}{R} \gamma_{i} \omega_{I J} D_{i} \tilde{C}^{J}+i \gamma_{3} \omega_{I J} \tilde{C}^{J}\left(\phi_{1}-\phi_{2}\right) \\
\delta A_{i}^{(1)}=\delta A_{i}^{(2)} & =-\frac{g}{2 \sqrt{2}}\left(\tilde{C}^{I} \tilde{\psi}^{J \dagger} \gamma_{i} \omega_{I J}+\omega^{I J} \gamma_{i} \tilde{\psi}_{I} \tilde{C}_{J}^{\dagger}\right) \\
\delta \phi_{1}=\delta \phi_{2} & =-\frac{R g}{2 \sqrt{2}}\left(\tilde{C}^{I} \tilde{\psi}^{J \dagger} \gamma_{3} \omega_{I J}+\omega^{I J} \gamma_{3} \tilde{\psi}_{I} \tilde{C}_{J}^{\dagger}\right) .
\end{aligned}
$$

However, if we were to solve for $\phi_{1}, \phi_{2}$, we would get

$$
\begin{aligned}
\delta \tilde{C}^{I} & =i \omega^{I J} \tilde{\psi}_{J} \\
\delta \tilde{\psi}_{I} & =-\gamma_{i} \omega_{I J} D_{i} \tilde{C}^{J}+i \gamma_{3} \omega_{I J} \tilde{C}^{J}\left[\frac{\sqrt{2}}{4 g} \frac{\epsilon^{i j} F_{i j}^{(1)}}{\tilde{C}^{I} \tilde{C}_{I}^{\dagger}}-\frac{1}{2 \tilde{C}^{I} \tilde{C}_{I}^{\dagger}}\left(\tilde{\psi}^{I \dagger} \gamma_{3} \psi_{I}\right)\right] \\
\delta A_{i}^{(1)} & =-\frac{g}{2 \sqrt{2}}\left(\tilde{C}^{I} \tilde{\psi}^{J \dagger} \gamma_{i} \omega_{I J}+\omega^{I J} \gamma_{i} \tilde{\psi}_{I} \tilde{C}_{J}^{\dagger}\right) .
\end{aligned}
$$

This is only an on-shell supersymmetry of the action with $\phi_{i}$ eliminated (specifically, on$A_{i}^{(1)}$-shell, i.e. using $(2.62)$ ), since for instance by varying the $\tilde{C}^{I}$ in $(2.61)$ we get unique 
terms involving $\left(\tilde{C}^{I} \tilde{C}_{I}^{\dagger}\right)^{-2}$, of the type

$$
-\frac{1}{R^{2}}\left(\tilde{C}^{I} \delta \tilde{C}_{I}^{\dagger}\right)\left[\frac{R \sqrt{2}}{4 g \tilde{v}} \frac{\epsilon^{i j} F_{i j}^{(1)}}{\tilde{C}^{I} \tilde{C}_{I}^{\dagger}}-\frac{R}{2 \tilde{C}^{I} \tilde{C}_{I}^{\dagger}}\left(\tilde{\psi}^{I \dagger} \gamma_{3} \psi_{I}\right)\right]^{2} .
$$

However, when using the $A_{i}^{(1)}$ equation of motion, the above becomes of similar type to other more conventional terms.

\section{Interpretational points}

We now move towards interpreting the dimensionally reduced ABJM action obtained in the previous section. This turns out to hide certain subtleties and involves an order of limits.

\subsection{From ABJM to BST formulation for the membrane}

Let us begin with the case of the single M2-brane in the Green-Schwarz-type supermembrane description of Bergshoeff-Sezgin-Townsend (BST) [2]. In a general supergravity background this is given by

$$
S=\int d^{3} x\left[\frac{1}{2} \sqrt{-g} g^{\mu \nu} E_{\mu}^{A} E_{\nu}^{B} \eta_{A B}+\frac{1}{2} \epsilon^{\mu \nu \lambda} E_{\mu}^{A} E_{\nu}^{B} E_{\lambda}^{C} C_{A B C}-\frac{1}{2} \sqrt{-g}\right],
$$

where

$$
E_{\mu}^{A}=\left(\partial_{\mu} Z^{M}\right) E_{M}^{A}
$$

In the above the $Z^{M}$ are superspace coordinates while $E_{M}^{A}$ is the supervielbein, such that

$$
g_{\mu \nu}=E_{\mu}^{A} E_{\nu}^{B} \eta_{A B}
$$

where $\mu=0,1,2$ are worldvolume while $A=0, \ldots, 10$ spacetime indices. The superfields satisfy the $11 \mathrm{~d}$ supergravity constraints. The bosonic degrees of freedom are $X^{A}$, which in static gauge reduce to the $C^{I}$ scalars, and involve no gauge fields.

At the same time, for the $N=1$ ABJM action of (2.56) and for $k=1$, one once again expects to obtain the static gauge action for a single supermembrane in flat space. Compared to the BST approach, this is a formulation that does involve auxiliary gauge fields.

Naturally, the formulations with and without gauge fields should be equivalent and this can be established as follows: ${ }^{6}$ Since the gauge fields are abelian, we can rewrite (2.56) in terms of their sum and difference

$$
\begin{aligned}
& Q_{\mu}=\left(A_{\mu}^{(1)}+A_{\mu}^{(2)}\right) \\
& B_{\mu}=\left(A_{\mu}^{(1)}-A_{\mu}^{(2)}\right),
\end{aligned}
$$

\footnotetext{
${ }^{6} \mathrm{~A}$ version of this procedure embedded in the nonabelian theory also appears in [19].
} 
obtaining

$$
S_{\mathrm{ABJM}}^{N=1}=\int d^{3} x\left[\frac{k}{4 \pi} \epsilon^{\mu \nu \lambda} B_{\mu} \partial_{\nu} Q_{\lambda}-i \psi^{I \dagger} \gamma^{\mu} D_{\mu} \psi_{I}-D_{\mu} C_{I}^{\dagger} D^{\mu} C^{I}\right]
$$

We next define the field strength $H_{\mu \nu}=\partial_{\mu} Q_{\nu}-\partial_{\nu} Q_{\mu}$ and treat it as an independent field. This is achieved by introducing a Lagrange multiplier that imposes the Bianchi identity on $H_{\mu \nu}$ through the equations of motion for the 'dual photon' $\sigma$ :

$$
S_{\mathrm{ABJM}}^{N=1}=\int d^{3} x\left[\frac{k}{8 \pi} \epsilon^{\mu \nu \lambda} B_{\mu} H_{\nu \lambda}+\frac{1}{8 \pi} \sigma \epsilon^{\mu \nu \lambda} \partial_{\mu} H_{\nu \lambda}-i \psi^{I \dagger} \gamma^{\mu} D_{\mu} \psi_{I}-D_{\mu} C_{I}^{\dagger} D^{\mu} C^{I}\right] .
$$

Integrating this new term by parts we find

$$
S_{\mathrm{ABJM}}^{N=1}=\int d^{3} x\left[\frac{k}{8 \pi} \epsilon^{\mu \nu \lambda} B_{\mu} H_{\nu \lambda}-\frac{1}{8 \pi} \epsilon^{\mu \nu \lambda}\left(\partial_{\mu} \sigma\right) H_{\nu \lambda}-i \psi^{I \dagger} \gamma^{\mu} D_{\mu} \psi_{I}-D_{\mu} C_{I}^{\dagger} D^{\mu} C^{I}\right] .
$$

It is now possible to integrate out $H_{\mu \nu}$, arriving at the relation

$$
B_{\mu}=\frac{1}{k} \partial_{\mu} \sigma
$$

with the $\mathrm{U}(1)_{B}$ gauge transformations acting on the dual photon as

$$
\sigma \rightarrow \sigma+k \theta
$$

Accordingly the covariant derivatives become

$$
D_{\mu} C^{I}=\partial_{\mu} C^{I}-i\left(A^{(1)}-A^{(2)}\right) C^{I}=\partial_{\mu} C^{I}-i B_{\mu} C^{I}=\partial_{\mu} C^{I}-\frac{i}{k} \partial_{\mu} \sigma C^{I}
$$

and the action can be rewritten in terms of a new set of matter fields

$$
\hat{C}^{I}=e^{-\frac{i \sigma}{k}} C^{I} \quad \text { and } \quad \hat{\psi}_{I}=e^{-\frac{i \sigma}{k}} \psi_{I}
$$

resulting in

$$
S_{\mathrm{ABJM}}^{N=1}=\int d^{3} x\left[-i \hat{\psi}^{I \dagger} \gamma^{\mu} \partial_{\mu} \hat{\psi}_{I}-\partial_{\mu} \hat{C}_{I}^{\dagger} \partial^{\mu} \hat{C}^{I}\right]
$$

In this manner the auxiliary gauge fields have been eliminated from the action. We note that as $\sigma$ is dual to the $\mathrm{U}(1)_{B}$ gauge field, it is a compact scalar with an associated periodic shift-symmetry.

However the above still transform under the $\mathrm{U}(1)_{B}$ gauge transformations, which we will next gauge-fix. In order to do so, we need to determine the periodicity of $\sigma$ which follows from a quantisation condition on the flux $H$. We have already defined $H=F^{(1)}+F^{(2)}$. By imposing the standard Dirac quantisation condition on the original gauge fields $\int d F^{(1,2)} \in$ $2 \pi \mathbb{Z}$, we get

$$
\int \frac{1}{2} \epsilon^{\mu \nu \lambda} \partial_{\mu} H_{\nu \lambda}=\int d H=\int d F^{(1)}+\int d F^{(2)} \in 4 \pi \mathbb{Z}
$$


and by plugging this into (3.6) and requiring that the path integral remains invariant under periodic shifts of $\sigma$, we determine that the latter has period $2 \pi$ [19]. This can be then used to gauge-fix the $\mathrm{U}(1)_{B}$ symmetry through (3.9) and set $\sigma=0 \bmod 2 \pi$. However, this periodicity imposes an additional identification on the $\mathrm{U}(1)$-invariant fields $\hat{C}^{I}, \hat{\psi}_{I}$

$$
\hat{Z}^{A} \cong e^{-\frac{2 \pi i}{k}} \hat{Z}^{A} \quad \text { and } \quad \hat{\psi}_{A} \cong e^{-\frac{2 \pi i}{k}} \hat{\psi}_{A}
$$

Therefore, we have obtained that (3.12) along with the above identification is nothing but the dynamical part for the action of a single BST M2-brane propagating on a $\mathbb{C}^{4} / \mathbb{Z}_{k}$ background. For the case of $k=1$, one recovers the action for a single membrane in flat space.

\subsection{From the reduced action to a Green-Schwarz string action}

Having established the relationship between the $N=1$ ABJM and BST actions, we now repeat the argument in the dimensionally reduced theory, which will exhibit some subtle points. As before, the reduced coordinate is denoted by $y$ and $i=0,1$ indicate the $2 \mathrm{~d}$ coordinates. Dimensionally reducing we get for $B_{\mu}$ and $Q_{\mu}{ }^{7}$

$$
\begin{aligned}
B_{y} & =\frac{1}{R}\left(\phi_{1}-\phi_{2}\right), & Q_{y} & =\frac{1}{R}\left(\phi_{1}+\phi_{2}\right) \\
B_{i} & =A_{i}^{(1)}-A_{i}^{(2)}, & Q_{i} & =A_{i}^{(1)}+A_{i}^{(2)} .
\end{aligned}
$$

Expressing the abelian CS piece of (2.56) in terms of $B$ and $Q$, we get

$$
S_{C S}=\frac{k}{4 \pi} \int d^{3} x \epsilon^{\mu \nu \lambda} B_{\mu} \partial_{\nu} Q_{\lambda} \rightarrow \frac{k R}{4 \pi} \int d^{2} x\left[B_{y} \epsilon^{i j} \partial_{i} Q_{j}+\epsilon^{i j} B_{i} \partial_{j} Q_{y}\right] .
$$

We define $H_{i j} \equiv 2 \partial_{[i} Q_{j]}$ but cannot treat it as an independent field, since it is not possible to introduce a Lagrange multiplier that imposes its Bianchi identity. On the other hand, we can obtain an independent field $H_{j y}$ by first defining $H_{j y}=\partial_{j} Q_{y}$ and then introducing it with a Lagrange multiplier $\tilde{\sigma}$. The equivalent $\mathrm{CS}$ action in $2 \mathrm{~d}$ is then

$$
S_{C S}=\frac{R}{8 \pi} \int d^{2} x\left[k B_{y} \epsilon^{i j} H_{i j}+k \epsilon^{i j} B_{i} H_{j y}+\tilde{\sigma} \epsilon^{i j} \partial_{i} H_{j y}\right] .
$$

The rest of the dimensionally reduced action is easily obtained from (2.6) in the abelian limit

$$
\int d^{2} x\left[-D_{i} \tilde{C}_{I}^{\dagger} D^{i} \tilde{C}^{I}-i \tilde{\psi}^{I \dagger} \gamma^{i} D_{i} \tilde{\psi}_{I}-\left(B_{y}\right)^{2} \tilde{C}^{I} \tilde{C}_{I}^{\dagger}-B_{y} \tilde{\psi}^{I \dagger} \gamma_{3} \psi_{I}\right]
$$

where the last two terms come from $D_{y} \sim-i B_{y}$ terms.

Now, if in the dimensionally reduced gauge action one integrates out $\phi_{1}, \phi_{2}$, that is $B_{y}$ and $Q_{y}$ in (3.16) plus (3.18), one obtains (2.58) with (2.61). But if we instead go to (3.17) and eliminate $H_{j y}$ we get

$$
B_{i}=\frac{1}{k} \partial_{i} \sigma
$$

\footnotetext{
${ }^{7}$ Note that in this subsection we define $B_{i}, Q_{i}$ without a prefactor of $\frac{1}{2}$.
} 
and then, as in the previous section,

$$
D_{i} \tilde{C}^{I} \rightarrow \partial_{i} \tilde{C}^{I}-\frac{i}{k} \partial_{i} \sigma \tilde{C}^{I}
$$

By eliminating $Q_{j}$

$$
\partial_{i} B_{y}=0 \Rightarrow B_{y}=c,
$$

where $c$ some constant. For the reader who might worry that $B_{y}=\frac{1}{R}\left(\phi_{1}-\phi_{2}\right)=c$ seems to contradict (2.60), we should note that it is consistent with (3.21) on-shell, since the equation of motion for $A^{(1)}$ implies

$$
\frac{\sqrt{2}}{4 g \tilde{v}} \epsilon^{i j} F_{i j}^{(1)}-\tilde{\psi}^{I \dagger} \gamma_{3} \tilde{\psi}_{I}=c \tilde{C}^{I} \tilde{C}_{I}^{\dagger}
$$

which is the same as (2.62).

Finally with the redefinitions

$$
\hat{\tilde{C}}^{I}=e^{-\frac{i \sigma}{k}} \tilde{C}^{I} ; \quad \hat{\tilde{\psi}}_{I}=e^{-\frac{i \sigma}{k}} \tilde{\psi}_{I}
$$

we arrive at the action

$$
S=\int d^{2} x\left[-\partial_{i} \hat{\tilde{C}}^{I} \partial^{i} \hat{\tilde{C}}_{I}^{\dagger}-i \hat{\tilde{\psi}}^{I \dagger} \gamma^{i} \partial_{i} \hat{\tilde{\psi}}_{I}-c^{2} \hat{\tilde{C}}^{I} \hat{\tilde{C}}_{I}^{\dagger}-c \hat{\tilde{\psi}}^{I \dagger} \gamma_{3} \hat{\tilde{\psi}}_{I}\right]
$$

which is a Green-Schwarz-type action plus some arbitrary mass terms. These can be put to zero by choosing $c=0$, since $c$ is an arbitrary constant at this point. We will come back to the interpretation of this constant soon.

\subsection{Interpretation of the $2 \mathrm{~d}$ action}

So far we have only discussed the set of algebraic steps that relate to the reduction process. We now turn our attention to assigning an interpretation to the resulting action.

\subsubsection{Intuition from String/M-theory}

As described in the introduction, the natural expectation is that reducing an action for M2-branes on a circle should lead to fundamental strings. We indeed discussed in Section 3.2 how the abelian, dimensionally-reduced ABJM action can be transformed to a GreenSchwarz action on an orbifold background. On the other hand, the presence of a single gauge field on the $2 \mathrm{~d}$ worldvolume before the transformation would naïvely suggest that it describes a D1-brane.

To further understand this, consider the case of M-theory on $T^{2}$. The compactification of a single M2-brane on the 2 circles (one transverse, $R_{2}$, one parallel, $R_{1}$ ) depends on the order of compactification: Indeed, if we compactify first on $R_{2}$, we get a D2-brane in $10 \mathrm{~d}$, with $g_{s}=\frac{R_{2}}{l_{s}}$. A further $R_{1}$ compactification must be followed by T-duality on $R_{1}$, to 
obtain a D1 in IIB, upon which $g_{s} \rightarrow g_{s} \frac{l_{s}}{R_{1}}=\frac{R_{2}}{R_{1}}$. On the other hand, by first compactifying on $R_{1}$ we get an F1 in $10 \mathrm{~d}$ but with $g_{s}=\frac{R_{1}}{l_{s}}$. By further compactifying on $R_{2}$ we must perform a T-duality in order to arrive at IIB, getting $g_{s}=\frac{R_{1}}{R_{2}}$. The two cases are related by $g_{s} \rightarrow \frac{1}{g_{s}}$, i.e. S-duality in $9 \mathrm{~d}$, which takes the fundamental string to a D1-brane.

However, the string coupling enters the two actions in a different way: For D1's it appears through $\left(g_{Y M}^{D 1}\right)^{2}=\frac{g_{s}}{2 \pi \alpha^{\prime}}$, in canonical normalisation, while for F1's as an additive term $\int d^{2} x \phi R^{(2)} \sim \ln g_{s} \chi$, with $\chi$ the Euler characteristic of the worldsheet. As a result one cannot make the duality precise at the level of the actions in their conventional formulations.

\subsubsection{Intuition from Higgsing}

We will gain some further insight into the physics of our action from looking at the Higgsed theory. As we have already seen, in the presence of a $\operatorname{VEV}\left\langle\tilde{C}^{I}\right\rangle=\tilde{v} \delta_{1}^{I} \mathbb{1}_{N \times N}$ the action (2.18) at general $N$ has a gauge coupling

$$
g \tilde{v}=\frac{2 \pi \sqrt{2} \tilde{v}}{k R} \equiv g_{Y M}^{D 1}
$$

Note that as far as $g_{Y M}^{D 1}$ is concerned, a finite coupling can be obtained by having $k$ and $\tilde{v}$ either be generic or both large, so one needs to look at other criteria. Furthermore, the coupling does not differentiate between a D1-brane in 9 or 10 dimensions.

By first considering the case of large $\tilde{v}$ and large $k$, we indeed obtained a D1-brane either in 9 or 10 dimensions, depending on the fields that were integrated out: By choosing $\phi_{1}$ and $\phi_{2}$, i.e. $\tilde{B}_{y}$ and $\tilde{Q}_{y}$, the compact scalar was eaten by the massive vector $B_{i}$ through a version of the ordinary Higgs mechanism, which was in turn replaced by a new scalar $\lambda$. For the T-dual version of the dimensionally reduced ABJM theory, $Q_{i}$ and the $\tilde{Q}_{y}=\phi_{1}+\phi_{2}$ combination were traded for the scalar corresponding to the compact dimension and became dynamical in the Higgsed theory, in the spirit of [4].

One needs to keep in mind that the compactification picture is valid at worldvolume energies $E \ll 1 / R$ and that a prospective D1-brane description would be weakly coupled if

$$
g_{\text {eff }}=\frac{g_{Y M}^{D 1}}{E}=2 \pi \sqrt{2} \frac{\tilde{v}}{k} \frac{1}{E R} \ll 1 .
$$

We see that this is only possible for small $\frac{\tilde{v}}{k}$, which matches with the intuition that we need to consider large $k$ to compactify a transverse scalar. We also need to be in an intermediate energy regime where

$$
(2 \pi \sqrt{2}) \frac{\tilde{v}}{k} \frac{1}{R} \ll E \ll \frac{1}{R} .
$$

At generic $k$ the above theory is strongly coupled. At generic $\tilde{v}$ there are $\mathcal{O}\left(\frac{1}{\tilde{v}}\right)$ corrections and the theory is not just SYM. However, in both these cases, one would instead expect an S-dual F1 description, at least if one eliminates $\phi_{i}$ as was done for $N=1$ in 
(2.58)-(2.61). From this angle, the fact that the action still has a worldvolume gauge field is not crucial, since we saw in Section 3.2 that it can be integrated out upon explicitly imposing the orbifold conditions on the matter fields to obtain a Green-Schwarz type action for the string.

\subsubsection{Reducing/T-dualising "M2 to D2"}

The Higgsed actions can also be obtained from the worldvolume reduction or T-duality of the ABJM membrane through the mechanism of [4], after giving a large VEV $v$ to one of the original ABJM worldvolume scalars. That is, we can consider Higgsing the theory before performing the dimensional reduction.

In terms of a geometric description the value of $k$ is interpreted as the rank of the $\mathbb{C}^{4} / \mathbb{Z}_{k}$ orbifold singularity on which the membranes are sitting. Going off to the Coulomb branch at large $v$ and $k$ results in type IIA String Theory dynamics. The M2 to D2 reduction is manifest in a way similar to the models of 'deconstruction' [20], by having a fixed and finite radius of compactification for the transverse direction. In fact, the large- $k$ dynamics are those of IIA even at the superconformal point (zero VEVs) where one has four complex scalars, as was made apparent by the analysis of [1], though of course at finite or zero VEV one probes an M-theory radius that varies between zero and a small nonzero value.

The resulting field theory action is $3 \mathrm{~d}$ SYM with corrections of order $\mathcal{O}\left(\frac{1}{v}\right)$ and gauge coupling

$$
g_{Y M}^{D 2}=\frac{2 \pi \sqrt{2} v}{k}=\frac{2 \pi \sqrt{2} \tilde{v}}{k \sqrt{R}},
$$

where the $11 \mathrm{~d}$ VEV $v$ is related to the $10 \mathrm{~d}$ one by $\tilde{v}=\sqrt{R} v$. On the other hand, at generic $k$ and $\tilde{v}$ one remains in the M2-brane regime.

Performing a worldvolume dimensional reduction/T-duality we again recover the D1action in $9 \mathrm{~d}$ and $10 \mathrm{~d}$ respectively. This is essentially because the Higgsing and reduction/Tduality operations commute. Our dimensionally reduced action is once more better thought of as one for a fundamental string, at least if the $\phi_{i}$ are eliminated, as we did for the $N=1$ case in $(2.58)-(2.61)$.

\subsubsection{Interpretation in terms of 'master' action}

We conclude that our general action Eq. (2.6), with all the auxiliary fields, contains information for all of the IIA F-string in 10d, its T- and S-dual IIB D-string in 10d, as well as the latter's T-dual IIA D-string in 9d (compactified D2-brane). The IIA F-string in 10d and the IIA D-string in $9 \mathrm{~d}$ are related through the compactification obtained by large $k$ together with an S-duality in 9d. Therefore we can think of the action (2.6) as a 'master' action that contains information about both T- and S-duality.

Even though we have recovered a precise field theoretic realisation of T-duality in Section 2.1.3, it is difficult to disentangle how the S-duality acts. It seems that the two 
components needed are the transformation of Section 3.2 and the transverse compactification through large $\tilde{v}$ and large $k$. We should also note that while the discussion made heavy use of the $N=1$ case, for which more explicit formulas were available, we still expect that at general $N$ the action (2.6) is a 'master' action for T- and S-duality.

\subsubsection{Understanding the F1-string interpretation}

There remains one point that has not yet been clarified for the 'master' action. If the equivalent action (3.24) is to have an F1 interpretation, it should describe a string moving in the orbifold background $\mathbb{C}^{4} / \mathbb{Z}_{k}$. As such, it must provide a natural explanation for the mass terms in (3.24). Indeed, note that $c$ has mass dimension 1, so we can instead denote it by a mass $\mu$.

Consider a straight string in $\mathbb{C}^{4}$ whose endpoints span exactly a $\frac{2 \pi}{k}$ angle from the origin. By making the $\mathbb{Z}_{k}$ identification we create a noncontractible string with winding number 1. This can however slide towards the origin by virtue of its tension, unlike the case of usual winding in a circle direction. Taking this string to be symmetric relative to the origin, its energy will be ${ }^{8}$

$$
E=\operatorname{Tr} 2 \sin \frac{\pi}{k},
$$

where $T=\frac{1}{2 \pi \alpha^{\prime}}$ is the string tension and $r$ is the radius from the origin of spacetime to the endpoints of the string, $r=|X(0)|$. The force pulling the string towards the origin will be

$$
F=\frac{d E}{d r}=2 T \sin \frac{\pi}{k} \equiv m \frac{d^{2} r}{d t^{2}}
$$

where $m=E=2 \operatorname{Tr} \sin \frac{\pi}{k}$ is the mass of the string. Substituting and introducing the appropriate sign we get

$$
\frac{d^{2} r}{d t^{2}}=-\frac{1}{r}=-\frac{1}{r^{2}} r \equiv-\mu^{2} r
$$

Here we have made assumption that due to its tension the string stays straight and symmetric as it slides towards the origin. Then the position $X(\sigma)$ along the string in some Cartesian spacetime reference system varies relative to the endpoints, i.e.

$$
\frac{\ddot{X}(\sigma)}{X(\sigma)}=\frac{\ddot{X}(0)}{X(0)}=-\mu^{2} .
$$

This matches the worldvolume equation of motion of the above straight string, $X^{\prime}=0$, with a worldvolume mass term $\mu$, namely

$$
\ddot{X}(\sigma)=-\mu^{2} X(\sigma) .
$$

Since $r$ is arbitrary then so is $\mu$, as was also the case in our worldvolume analysis. Note that we have not needed any approximation for this result. Indeed, the equations of motion

\footnotetext{
${ }^{8}$ If the string is asymmetric relative to the origin its tension will make it symmetric since that corresponds to a minimum length for a given center-of mass distance to the origin.
} 
for $\mu$ were $\partial_{i}(\mu)=0$, that is $\mu$ constant on the worldvolume, and this is what we find. Of course $\mu=\frac{1}{r}$, with $r$ the radius at the endpoint, so $\mu$ depends on the spacetime boundary of the string but that does not contradict our constraints.

To conclude, the arbitrary scalar mass term in (3.24) is just the effect of having a string in $\mathbb{C}^{4} / \mathbb{Z}_{k}$. The fermion mass term is understood as the necessary supersymmetric extension. This completes our understanding of the $N=12$ d action as an F-string in $\mathbb{C}^{4} / \mathbb{Z}_{k}$

\section{Conclusions}

In this note we performed the dimensional reduction of the ABJM model, studying the resulting action and its physical interpretation. The reduced action includes a set of auxiliary gauge and scalar fields. Focusing on the Coulomb branch of the theory, we found that for a large $\mathrm{VEV} \tilde{v}$, in the regime where $k$ was large, one obtains either the action of $N$ D1-branes in 9d (compactified D2-branes) or the T-dual action of $N$ D1-branes in 10d, depending on which combination of auxiliary fields are integrated out. The two actions were related by a field theoretic T-duality transformation, following [17].

For the special case of $N=1$, at an arbitrary VEV $\tilde{v}$ and at arbitrary $k$, we showed that the equivalence in $3 \mathrm{~d}$ between the $N=1 \mathrm{ABJM}$ model and the BST action on $\mathbb{C}^{4} / \mathbb{Z}_{k}$ can be reduced to give a Green-Schwarz string moving in $\mathbb{C}^{4} / \mathbb{Z}_{k}$. This led us to propose that the dimensionally reduced action can be thought of as a 'master' action encoding information about both T- and S-duality. The field theoretic realisation of S-duality in the nonabelian case remains mysterious as ever and warrants further investigation. Since at generic $k / \mathrm{VEV}$ $\tilde{v}$ the $9 \mathrm{~d}$ D1-brane action obtained by eliminating $\phi_{i}$ is strongly-coupled/receives $\mathcal{O}\left(\frac{1}{\tilde{v}}\right)$ corrections, we suggest that it should instead be better thought of as a multiple F-string action.

\section{Acknowledgements}

We would like to thank Dario Martelli for discussions and comments. CP is supported by the STFC grant ST/G000395/1.

\section{References}

[1] O. Aharony, O. Bergman, D. L. Jafferis, and J. Maldacena, "N=6 superconformal Chern-Simons-matter theories, M2-branes and their gravity duals," JHEP 10 (2008) 091, arXiv:0806.1218 [hep-th].

[2] E. Bergshoeff, E. Sezgin, and P. K. Townsend, "Supermembranes and eleven-dimensional supergravity," Phys. Lett. B189 (1987) 75-78. 
[3] M. J. Duff, P. S. Howe, T. Inami, and K. S. Stelle, "Superstrings in D = 10 from supermembranes in D = 11," Phys. Lett. B191 (1987) 70.

[4] S. Mukhi and C. Papageorgakis, "M2 to D2," JHEP 05 (2008) 085, arXiv:0803.3218 [hep-th].

[5] J. Distler, S. Mukhi, C. Papageorgakis, and M. Van Raamsdonk, "M2-branes on M-folds," JHEP 05 (2008) 038, arXiv:0804.1256 [hep-th].

[6] M. A. Santos and I. V. Vancea, "Two Dimensional Effective Field Theory from Bagger- Lambert-Gustavsson Model," Mod. Phys. Lett. A24 (2009) 2275-2284, arXiv:0809.0256 [hep-th].

[7] P. Franche, "Reduction of the $\mathrm{N}=8 \mathrm{BLG}$ and $\mathrm{N}=6 \mathrm{BL}$ Theories to 2D Effective Field Theories," arXiv:0811.1443 [hep-th].

[8] J. Bagger and N. Lambert, "Gauge Symmetry and Supersymmetry of Multiple M2-Branes," Phys. Rev. D77 (2008) 065008, arXiv:0711.0955 [hep-th].

[9] A. Gustavsson, "Algebraic structures on parallel M2-branes," Nucl. Phys. B811 (2009) 66-76, arXiv:0709.1260 [hep-th].

[10] K. Hosomichi, K.-M. Lee, S. Lee, S. Lee, and J. Park, "N=5,6 Superconformal Chern-Simons Theories and M2-branes on Orbifolds," JHEP 09 (2008) 002, arXiv:0806.4977 [hep-th].

[11] J. Gomis, D. Rodriguez-Gomez, M. Van Raamsdonk, and H. Verlinde, "A Massive Study of M2-brane Proposals," JHEP 09 (2008) 113, arXiv:0807.1074 [hep-th].

[12] H. Nastase, C. Papageorgakis, and S. Ramgoolam, "The fuzzy $S^{2}$ structure of M2-M5 systems in ABJM membrane theories," JHEP 05 (2009) 123, arXiv:0903.3966 [hep-th].

[13] H. Nastase and C. Papageorgakis, "Fuzzy Killing Spinors and Supersymmetric D4 action on the Fuzzy 2-sphere from the ABJM Model," JHEP 12 (2009) 049, arXiv:0908.3263 [hep-th].

[14] H. Nastase and C. Papageorgakis, "Bifundamental Fuzzy 2-Sphere and Fuzzy Killing Spinors," SIGMA 6 (2010) 058, arXiv:1003.5590 [math-ph].

[15] Y. Pang and T. Wang, "From N M2's to N D2's," Phys. Rev. D78 (2008) 125007, arXiv:0807.1444 [hep-th].

[16] X. Chu, H. Nastase, B. E. Nilsson, and C. Papageorgakis, "Higgsing M2 to D2 with gravity: $\mathrm{N}=6$ chiral supergravity from topologically gauged ABJM theory," arXiv:1012.5969 [hep-th]. 
[17] T. H. Buscher, "A Symmetry of the String Background Field Equations," Phys. Lett. B194 (1987) 59.

[18] S. Sasaki, "On Non-linear Action for Gauged M2-brane," JHEP 02 (2010) 039, arXiv:0912.0903 [hep-th].

[19] N. Lambert and C. Papageorgakis, "Relating $\mathrm{U}(\mathrm{N}) \mathrm{xU}(\mathrm{N})$ to $\mathrm{SU}(\mathrm{N}) \mathrm{xSU}(\mathrm{N})$ Chern-Simons Membrane theories," JHEP 04 (2010) 104, arXiv:1001.4779 [hep-th].

[20] N. Arkani-Hamed, A. G. Cohen, D. B. Kaplan, A. Karch, and L. Motl, "Deconstructing (2,0) and little string theories," JHEP 01 (2003) 083, arXiv:hep-th/0110146. 